\title{
A Female Patient with Intraorbital Varix: A Case Report
}

\author{
Tahereh Darvishpour Kakhki ${ }^{1,{ }^{*}}$, Hojat Darvishpour Kakhki ${ }^{2}$ and Ghedsiyeh Azarkar ${ }^{3}$ \\ ${ }^{1}$ Ophthalmic Research Center, Shahid Beheshti University of Medical Sciences, Tehran, Iran \\ ${ }^{2}$ Department of Orthodontics, Tehran University of Medical Sciences, Tehran, Iran \\ ${ }^{3}$ Birjand Infectious Disease Research Center, Birjand University of Medical Sciences, Birjand, Iran \\ "Corresponding author: Ophthalmic Research Center, Shahid Beheshti University of Medical Sciences, Tehran, Iran. Email: t.darvishpor@yahoo.com
}

Received 2019 January 16; Revised 2019 June 26; Accepted 2019 July 16.

\begin{abstract}
The present study reported the case of a 23-year-old female patient with right-sided enophthalmos. After several episodes of spontaneous periorbital ecchymosis, she developed positional proptosis. The ophthalmologic examination was normal, except a 3-mm enophthalmos measured using the Naugle exophthalmometer, which improved significantly by the Valsalva maneuvers. The diagnostic imaging tests in orbital disease consisting of orbital computed tomography and orbital magnetic resonance imaging showed a retro-orbital vascular lesion. The most likely diagnosis was orbital varix, which is a rare orbital vascular lesion. It was decided to manage the patient conservatively, in the absence of complications such as proptosis with corneal exposure, optic nerve compression, or unacceptable appearance.
\end{abstract}

Keywords: Enophthalmos, Varix, Orbite

\section{Introduction}

The enophthalmos is a rare clinical feature usually occurring as a result of trauma (1).

Enophthalmos is the recession of the globe within the orbit causing congenital or traumatic orbital wall abnormalities, atrophy of the orbital contents (e g, radiotherapy, scleroderma, and chronic eye poking in blind infants orbital varices) or sclerosis (e g, metastatic scirrhous carcinoma and sclerosing orbital inflammatory disease) (2).

Varix is a dilation of one or more veins (3). In the orbit, a varix can become sizable to produce a mass effect. There is a considerable overlap among the orbital venous malformations such as orbital varix, varicocele, and venous angioma, and they probably represent a spectrum (4). Furthermore, there is a controversy whether varix and lymphangioma represent the same entity. It is observed that orbital varices are associated with the defects in orbital bone and cephaloceles, and that the inflammation of a thrombosed varix could predispose to meningitis in such cases (3).

In most of the cases, the unilateral and upper nasal quadrant is the favored site. This lesion generally involves the superior ophthalmic vein. Clinically, it is usually diag- nosed within the age range of 10 - 30 years characterized by the visible lesions in the eyelid or conjunctiva, or manifested in a form of positional proptosis following the Valsalva maneuver. Since the orbital venous channels are devoid of valves, the occurrence of a reversible proptosis is probable (5). The varices may rarely threaten the vision through optic nerve compression caused by acute hemorrhage or thrombosis. Chronic lesions may present as enophthalmos (6). Imaging modalities used in this regard include computed tomography (CT) scan, Doppler ultrasonography, and angiography. Doppler demonstrates the flow of blood. Rapid spiral CT during Valsalva maneuver shows characteristic enlargement of the engorged varix. Uniform contrast enhancement is observed. Sometimes phleboliths may also be seen. Angiography shows connection of the lesion to the venous system and completely fills the up-following injection. Management is usually conservative, but in the presence of signs of optic nerve compression, surgical removal is considered. Complete removal is usually not possible since the lesions are friable and unencapsulated, and bleed easily. Embolization using coils through a distal vein is another method to diminish the symptoms (5). 


\section{Case Presentation}

A 23-year-old female patient referred to the clinic in May 2016, complaining about a four-year history of painless positional proptosis of right eye as well as a history of two episodes of spontaneously right periorbital ecchymosis with no family history and no history of trauma.

The physical examination was normal, but in the ophthalmologic examination, a 3-mm enophthalmos was observed, which improved significantly by the Valsalva maneuvers (Figure 1). The visual acuity was 10/10. The pupillary reaction was normal. The intraocular pressure was in the normal limit $(11 \mathrm{mmHg}$ ). Examination of the anterior and posterior segments of the eye had normal results. A plain CT scan and MRI magnetic resonance imaging of the orbit revealed an abnormal vascular tortuosity in the posterior and inferomedial aspect of right eye globe, as well as a slight obliteration of the retrobulbar fat. No displacement of the optic nerve was observed. An orbital MRI with contrast showed an irregular mass located in the posterior and inferomedial aspect of the right eye globe (Figure 2). The differential diagnosis consisted of orbital varice, arteriovenous malformation, and lymphangioma, but the most likely diagnosis was orbital varice. Due to the diffuse nature of the lesion, absence of complications such as proptosis with corneal exposure, optic nerve compression, or unacceptable appearance, conservative management was adopted.

\section{Discussion}

The orbital varix is a rare orbital vascular lesion accounting for $0 \%-1.3 \%$ of all histopathologically proven orbital tumors (4).

Pathogenesis of the lesion is unclear, but the weakening of the vein wall may be the first occurrence of orbital varicose veins. On the other hand, orbital veins do not have a valve increasing the accumulation of blood in the orbit and thrombosis occurrence. The slow flow of blood causes dilation of the vein in the beginning, consequently creating a great varix $(1-4,6)$.

Venous malformations of the orbit (also known as orbital varices) are low-flow vascular lesions resulting from vascular dysgenesis. Patients may exhibit enophthalmos at rest, when the lesion is not engorged. The increase in the severity of the proptosis occurring when the patient's head is dependent or after performing the Valsalva maneuver suggests the presence of a venous malformation. The diagnosis can be confirmed via contrast-enhanced rapid spiral CT during the administration of Valsalva maneuver (or other approaches used for decreasing the venous return) showing characteristic enlargement of the engorged veins. Phleboliths may be present on imaging (5-8).

In the current study, a case was reported, where an orbital varix caused the positional proptosis. Since the orbital varix is connected to the systemic venous circulation, the proptosis is exacerbated as a result of performing the Valsalva maneuver and with prostrate.

Thus, the patients may exhibit enophthalmos at rest, when the lesion is not engorged. The diagnosis was confirmed through the use of contrast-enhanced orbital MRI during the administration of the Valsalva maneuver showing characteristic enlargement of the engorged veins.

Treatment is usually conservative. Biopsy should be avoided due to the risk of hemorrhage. Surgery is reserved for relief of significant pain or for cases in which the venous malformation causes vision threatening compressive optic neuropathy. Complete surgical excision is difficult, as these lesions often intertwine with normal orbital structures and directly communicate with the abundant venous reservoir in the cavernous sinus. Intraoperative embolization of the lesion may be helpful in the surgical removal. Embolization using the coils inserted through a distal venous cut-down is also reported to diminish the symptoms (6). Herein, it was decided to manage the patient conservatively in the absence of complications such as proptosis with corneal exposure, optic nerve compression, or unacceptable appearance.

\section{Footnotes}

Authors' Contribution: Study concept: Tahereh Darvishpour Kakhki, study design: Ghodsieh Azarkar and Hojat Darvishpour Kakhki.

Conflict of Interests: Authors declared no conflict of interest.

Ethical Approval: The current study was conducted with the consent of the patient under the supervision of Birjand University of Medical Sciences.

Funding/Support: There was no grant for the study. 

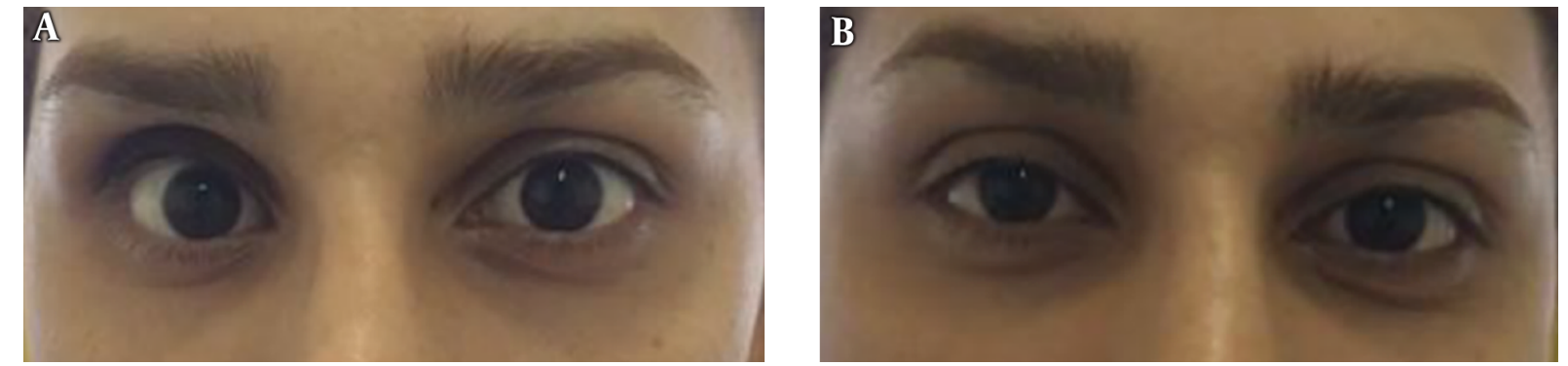

Figure 1. Enophthalmos of the right eye (B) improved significantly by the Valsalva maneuvers (A). *The photo was taken with the consent of the patient.
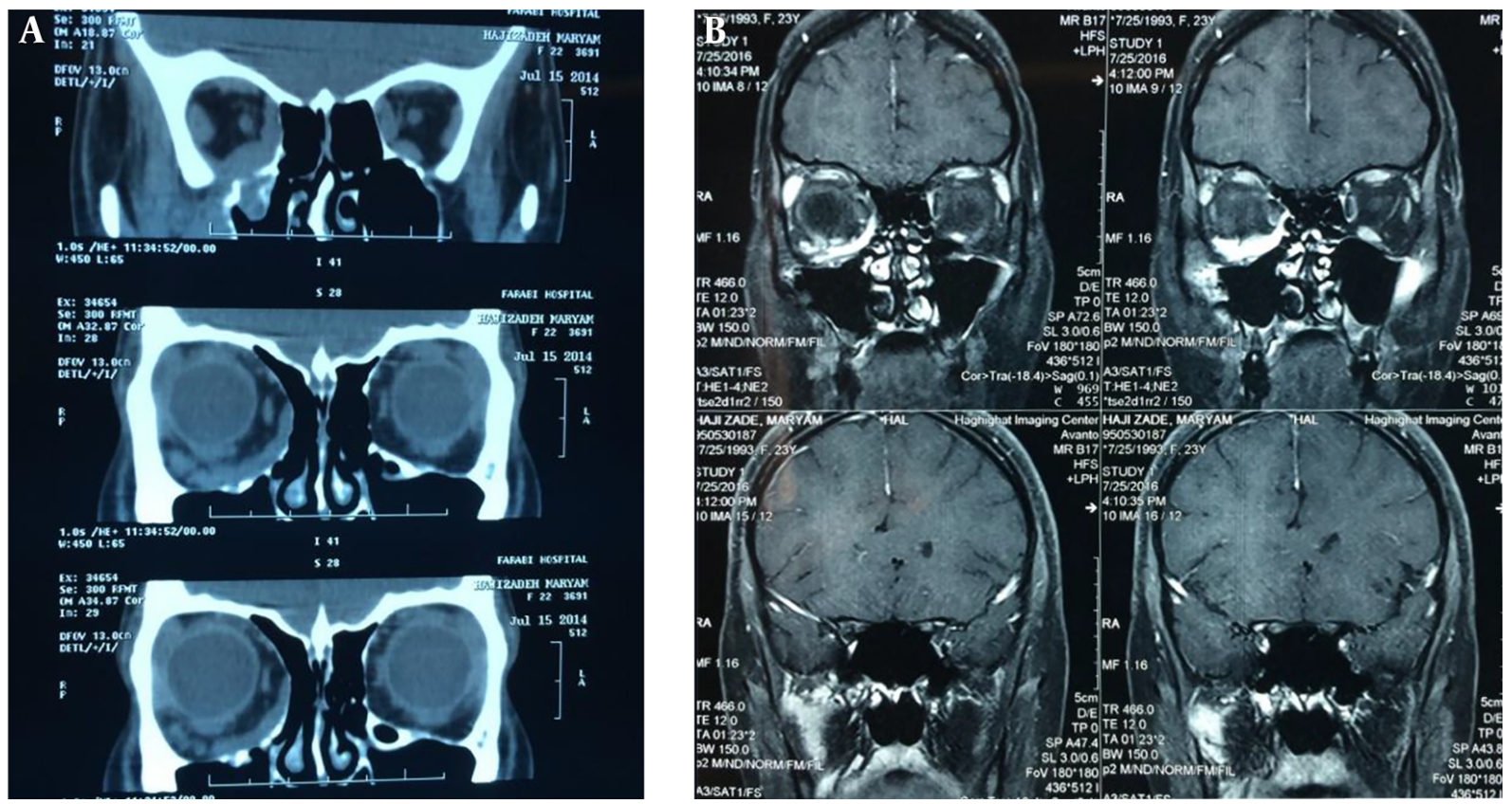

Figure 2. Plain computed tomography scan of the orbits (A) showing a soft tissue mass in the inferomedial orbit; magnetic resonance imaging of the orbits with contrast (B) showing homogenous enhancement of the soft tissue mass.

\section{References}

1. Smith RJ, Balbo NS, Lahoz Ibaceta MJ. [Enophthalmos secondary to an orbital varix]. Medicina (B Aires). 2014;74(2):127-9. Spanish. [PubMed: 24736258].

2. Bowling B. Kanski's clinical ophthalmology: A systematic approach. 8th ed. Orbit; 2016. p. 77-117.

3. Islam N, Mireskandari K, Burton BJ, Rose GE. Orbital varices, cranial defects, and encephaloceles: An unrecognized association. Ophthalmology. 2004;111(6):1244-7. doi: 10.1016/j.ophtha.2003.10.022. [PubMed: 15177979].

4. Karcioglu ZA. Orbital tumor: Diagnosis and treatment. 2nd ed. Springer Science \& Business Media; 2015. p. 154-73.
5. Wright JE, Sullivan T], Garner A, Wulc AE, Moseley IF. Orbital venous anomalies. Ophthalmology. 1997;104(6):905-13. doi: 10.1016/s01616420(97)30208-5. [PubMed: 9186428].

6. American Academy of Ophthalmology. Orbit, eyelids, and lacrimal system. European Board of Ophthalmology subcommittee; 2017.

7. Kim JW, Stewart WB, Spencer WH, Ellis DS. Acute expansion of an orbital vascular malformation. Arch Ophthalmol. 1999;117(6):844-5. doi: 10.1001/archopht.117.6.844.

8. Rubin PA, Remulla HD. Orbital venous anomalies demonstrated by spiral computed tomography. Ophthalmology. 1997;104(9):1463-70. doi: 10.1016/s0161-6420(97)30115-8. [PubMed: 9307642]. 